\title{
Brain Natriuretic Peptide and Left Ventricular Dysfunction in Chagasic Cardiomyopathy
}

\author{
Andre Talvani/ ${ }^{++}$, Manoel OC Rocha*, John Cogan** , Param Maewal**, \\ James de Lemos**, Antonio LP Ribeiro* , Mauro M Teixeira/ ${ }^{+}$
}

\begin{abstract}
Departamento de Bioquímica e Imunologia, Instituto Ciências Biológicas *Pós-Graduação em Medicina Tropical, Departamento de Clínica Médica, Faculdade de Medicina, Universidade Federal de Minas Gerais, Av. Antônio Carlos 6627, 31270-901, Belo

Horizonte, MG, Brasil **Universtiy of Texas Southwestern Medical Center, Dallas, TX, US
\end{abstract}

\begin{abstract}
Global left ventricular ( $L V)$ systolic dysfunction is the strongest predictor of morbidity and mortality in Chagas disease. Echocardiography is considered the gold standard for the detection of LV dysfunction, but not always available in endemic areas where chagasic cardiomyopathy is most common. Brain natriuretic peptide (BNP) is a neurohormone that has been recently described as a simple and inexpensive diagnostic and prognostic marker for patients with congestive heart failure. Chagasic patients $(n=63)$ and non-infected healthy individuals $(n=18)$ were recruited prospectively and underwent complete clinical examination, echocardiography and 24-h Holter monitoring. BNP was measured from thawed plasma samples using the Triage BNP test. We observed high levels of $B N P$ in association with depression of $L V$ ejection fraction, with increase of $L V$ end-diastolic diameter and with $L V$ premature complexes. An elevated concentration of BNP, defined as a concentration of $60 \mathrm{pg} / \mathrm{ml}$ or more, had a sensitivity of $91.7 \%$, specificity of $82.8 \%$, positive predictive value of $52.4 \%$, and negative predictive value of $98 \%$ for detecting $L V$ dysfunction ( $L V$ ejection fraction $<40 \%$ ). BNP measurement using a simple, relatively inexpensive and rapid test has a promising role in identifying LV dysfunction associated with chagasic cardiomyopathy. Equally important, patients with Trypanosoma cruzi infection who have low levels of BNP level in plasma have a very low likelihood of severe cardiac involvement, and echocardiography is probably not necessary.
\end{abstract}

Key words: brain natriuretic peptide - chagasic cardiomyopathy - left ventricular dysfunction - Chagas disease

Brain natriuretic peptide (BNP) is a neurohormone of cardiac origin that has been recently described as a diagnostic and prognostic marker for patients with congestive heart failure (Clerico et al. 2000, Cheng et al. 2001, Dao et al. 2001, McCullough et al. 2002, de Lemos et al. 2003). This peptide is synthesized and stored mainly in cardiac ventricles. Increased synthesis and release of BNP occur in response to ventricular volume expansion and pressure overload (Silberbach \& Roberts 2001). Functionally, it promotes diuresis, natriuresis, peripheral vasodilatation, renin angiotensin-aldosterone system inhibition and sympathetic nervous system inhibition and ameliorates some clinical manifestations caused by heart failure regardless of origin (Kuster et al. 2003).

Heart disease secondary to a progressive and chronic myocarditis is the most important clinical manifestation of Trypanosoma cruzi infection, the causative agent of

Financial support: Fapemig, the UNDP/World Bank/WHO Special Programme for Research and Training in Tropical Diseases (970728), CNPq

${ }^{+}$Corresponding author. Fax: +55-31-3499.2651. E-mail: mmtex@icb.ufmg.br

${ }^{++}$Present address: Instituto de Ciências Biológicas e da Saúde, Pontifícia Universidade Católica de Minas Gerais, Belo Horizonte, MG, Brasil

Received 27 April 2004

Accepted 18 August 2004
Chagas disease. Chronic chagasic cardiomyopathy (CCC) is characterized by a wide variety of anatomical and electrophysiological alterations that are accompanied by frequent atrial and ventricular arrhythmias. The severity of ventricular arrhythmias tends to correlate with the degree of left ventricular dysfunction (Rocha et al. 2003). Indeed, global left ventricular systolic dysfunction is the strongest predictor of morbidity and mortality in Chagas disease (Carrasco et al. 1994, Mady et al. 1994). Early diagnosis of systolic dysfunction may identify patients who would benefit from aggressive medical therapy.

Echocardiography is commonly used in the noninvasive assessment of chagasic cardiomyopathy (Bestetti 2001, Perez et al. 2003, Rocha et al. 2003). However, due to the relative high cost of the equipment and trained personnel involved, alternative low cost methods are desirable to diagnose ventricular dysfunction in chagasic patients. Recently, our group demonstrated that an elevation of BNP concentration in blood was a reliable indicator of systolic left ventricular dysfunction. In patients with abnormal electrocardiogram (ECG) and/or chest XRay, BNP elevation had a positive predictive value of $80 \%$ and a negative predictive value of $97 \%$ to diagnose left ventricular dysfunction (Ribeiro et al. 2002b). The present study had two main objectives. First, we sought to evaluate the relationship between plasma concentrations of BNP and left ventricular ejection fraction, left ventricular diastolic diameter and the incidence of ventricular premature complexes in $24 \mathrm{~h}$. Second, we aimed at confirming the ability of BNP to diagnose ventricular dysfunction in patients with chagasic heart disease. 


\section{MATERIALS AND METHODS}

Patients and study design - Studies described here received ethical clearance from the Institutional Review Board of Universidade Federal de Minas Gerais. The diagnosis of Chagas disease was based on the presence of at least two positive serological examinations using distinct techniques (ELISA, indirect hemaglutination or indirect immunofluorescence) in an individual with a relevant epidemiological history. Non-infected individuals had three negative serological examinations. All individuals were recruited and evaluated clinically at the Center for Reference and Treatment of Infectious Diseases (CTRDIP) at Hospital das Clinicas, Universidade Federal de Minas Gerais, where they underwent a complete clinical examination and performed the following laboratory workup: complete blood count, free T4, TSH, glucose, potassium, creatinine, blood urea nitrogen, electrocardiogram, chest X-ray, a $24 \mathrm{~h}$ Holter examination and echocardiography. Patients with hypertension, diabetes, thyroid or renal disturbances or any other cardiac or systemic diseases and those using steroidal drugs were excluded from this study.

The left ventricular ejection fraction (LVEF) was obtained through Simpson's method using the software provided with the equipment by an observer blinded to the clinical status (Barros et al. 2003). Twenty-four-hour Holter monitoring was performed using a portable three-channel cassette tape recorder (Dynamis, Cardios, São Paulo, Brazil) (Ribeiro et al. 2002a). Patients were encouraged to continue with their normal everyday activities during the recordings, with the avoidance of physical exercise or drugs that could interfere with autonomic function. Analysis of the tapes was performed when at least $18 \mathrm{~h}$ of high quality tracings were available. The recordings were analyzed on a Burdick/DMI Hospital Holter System (Spacelabs Burdick, Deerfield, Wisconsin) by a semi-automatic technique. Minimal means and maximal heart rate, the number of ectopic ventricular and supraventricular beats, the occurrence of pauses and heart blocks were recorded. In this study, ventricular tachyarrhythmia (see Fig. 3) was defined as the occurrence of one or more episodes of ventricular tachycardia during $24 \mathrm{~h}$ Holter monitoring.

Chagasic patients were prospectively categorized into two groups according to the degree of heart dysfunction: mild CCC and severe CCC. This classification was made by investigators blinded to results of BNP testing. Patients were classified as mild CCC $(n=23)$ if they had a normal EKG and chest X-ray and no more than minor alterations in their echocardiogram (eg. regional wall motion abnormalities). These patients would be classified as indeterminate form or $\mathrm{CCC} 1$, respectively, according to previously published clinical criteria from our group (Rocha et al. 2003). Patients with severe CCC $(n=40)$ were those with severe conduction defects (i.e. left bundle branch block, left anterior branch hemiblock with right bundle branch block or total atrioventricular block) or ventricular enlargement, as observed on the echocardiogram. The latter patients would be classified as $\mathrm{CCC} 4$ or CCC5, respectively, according to previously published clinical criteria from our group (Rocha et al. 2003). The control group consisted of 18 non-infected healthy individuals (Table I).

In order to confirm our previous study, chagasic patients were classified as having significant left ventricular systolic dysfunction if LVEF was $\leq 0.40$. This cutoff point was chosen since it has been previously used in other clinical trials performed in patients with $\mathrm{LV}$ dysfunction (Ribeiro et al. 2002a).

Measurement of BNP - A sample of blood (5 ml) was collected by a trained technician into tubes containing potassium EDTA ( $1 \mathrm{mg} / \mathrm{ml}$ of blood). Samples were centrifuged ( $3000 \mathrm{~g}$ for $10 \mathrm{~min}$ ) and plasma obtained and frozen at $-80^{\circ} \mathrm{C}$ until further analysis. Frozen specimens were shipped to UT Southwestern Medical Center (Dallas, TX) where BNP was measured from thawed samples using the Triage BNP test (Biosite Inc., US), by investigators blinded to clinical data. The Triage BNP test is a fluorescence immunoassay for the quantitative determination of BNP in plasma specimens. Precision, analytical sensitivity and stability characteristics of the system have been previously described (McCullough et al. 2002). The measurable range of the BNP assay was 5.0 to $1300.0 \mathrm{pg} / \mathrm{ml}$.

Statistical analysis - Data were expressed as mean \pm standard error or proportions and were compared among groups using ANOVA with Fisher multiple comparisons procedure or the Chi square test for $2 \mathrm{x} \mathrm{k}$ contingency tables, with Bonferroni correction. Logarithm transformation of non-normal or heteroscedatic data was performed to allow subsequent analysis. Pearson's coefficients were used to measure correlations between variables controlled, when necessary, for the effects of one or more additional variables. Linear regression analysis was performed to describe relationship between variables further. A $p$ value $<0.05$ was considered significant. The diagnostic performance of the BNP values in the diagnosis of LV systolic dysfunction in the Chagas disease population was evaluated using the receiver-operator-characteristic (ROC) curve, obtained using the software Analyse-it (Leeds, England). In brief, the ROC curve is a plot of the sensitivity of a test versus its false-positive rate ( 1 - specificity) for all possible cut points. The area under the curve, ranging from ' 0 ' to ' 1 ', provides a measure of the overall diagnostic accuracy of the test, that is, the ability of the test to correctly classify those with and without the disease. The other analyses were performed using statistical programs PRISM (GraphPad, San Diego, CA, US) and SPSS 10.0 (Chicago, IL, US).

\section{RESULTS}

The clinical characteristics of chagasic patients and non-infected individuals are shown in Table I. There was no significant difference in the sex distribution between the groups but non-infected individuals were slightly younger than patients with mild disease. Nevertheless, individuals infected with $T$. cruzi and with the mild form of CCC had essentially similar functional cardiovascular parameters as non-infected individuals. Patients with severe CCC had marked cardiovascular dysfunction with higher New York Heart Association (NYHA) classification, lower ejection fraction, greater left ventricular diameter and frequent presence of ventricular premature complexes in $24 \mathrm{~h}$ (Table I). 
TABLE I

Baseline characteristics of non-infected individuals and patients with chronic chagasic cardiomyopathy (CCC) with the mild and severe forms of the disease

\begin{tabular}{lccc}
\hline & $\begin{array}{c}\text { Non-infected } \\
(\mathrm{n}=18)\end{array}$ & $\begin{array}{c}\text { Mild } \\
(\mathrm{n}=23)\end{array}$ & $\begin{array}{c}\text { Severe } \\
(\mathrm{n}=40)\end{array}$ \\
Age & $36.3 \pm 11.0$ & $47.0 \pm 8.6^{a}$ & $42.2 \pm 10.0$ \\
\hline Sex (M) & $72 \%$ & $48 \%$ & $63 \%$ \\
LVEF (\%) & $68.0 \pm 5.9$ & $64.7 \pm 3.8$ & $49.0 \pm 13.1^{a, b}$ \\
LVDD (mm) & $48.8 \pm 4.6$ & $49.9 \pm 3.4$ & $58.2 \pm 7.6^{a, b}$ \\
NVPC in 24 h & ND & $87 \pm 264$ & $617 \pm 4198^{b}$ \\
BNP (pg/ml) & $6.7 \pm 6.3$ & $17.0 \pm 19.6^{a}$ & $117.7 \pm 208.3^{a, b}$ \\
\hline
\end{tabular}

Values are shown as mean \pm SD. LVEF: left ventricle ejection fraction; LVDD: left ventricle diastolic diameter; NVPC: number of ventricular premature complexes in $24 \mathrm{~h} ; a$ : $\mathrm{P}<0.01$ when compared to non-infected; $b: \mathrm{P}<0.01$ when compared to mild CCC. Non-normally distributed data were transformed before performing ANOVA and means comparisons.

The concentrations of BNP in plasma of chagasic and non-infected individuals distributed according to the functional NYHA classification is shown in Fig. 1A. There is a clear increase in BNP concentrations with the worsening of functional capacity. BNP levels were lowest among noninfected individuals, intermediate among those with mild CCC and highest among patients with severe infection (Fig. 1B). Of note, the majority (85\%) of patients with severe CCC were NYHA class I or II. Plasma BNP was also correlated with left ventricular ejection fraction, the left ventricle diameter and the number of ventricular premature complexes at $24 \mathrm{~h}$ (Table II).

The plasma concentration of BNP in patients with apical aneurysm and no episodes of non-sustained ventricular tachycardia was similar to that of non-infected individuals (Fig. 2). However, BNP concentrations were elevated among patients with apical aneurysm and nonsustained ventricular tachycardia (Fig. 2).

Fig. 3 shows the ROC curve for various concentrations of BNP in the diagnosis of LV dysfunction in the Chagas disease population: the area under the curve was $0.92 \pm 0.03$, indicating an excellent diagnostic performance.

\section{TABLE II}

Correlation of the concentration of brain natriuretic peptide in plasma and indices of ventricular function in patients with Chagas disease

\begin{tabular}{lcc}
\hline & $\mathrm{R}$ & $\mathrm{P}$ \\
\hline Log BNP x LVEF & 0.64 & 0.0001 \\
Log BNP x LVDD & 0.58 & 0.0001 \\
Log BNP x Log NVPC & 0.63 & 0.0001 \\
\hline
\end{tabular}

There were 23 and 40 patients with the mild or severe forms, respectively, of chronic chagasic cardiomyopathy. LVEF: left ventricle ejection fraction (\%); LVDD: left ventricle diastolic diameter $(\mathrm{mm})$; NVPC: number of ventricular premature complexes in $24 \mathrm{~h}$
An elevated concentration of BNP, defined as concentrations of $60 \mathrm{pg} / \mathrm{ml}$ or more, had a sensitivity of $91.7 \%$, specificity of $82.8 \%$, positive predictive value of $52.4 \%$ and negative predictive value of $98 \%$ for detecting LV dysfunction.

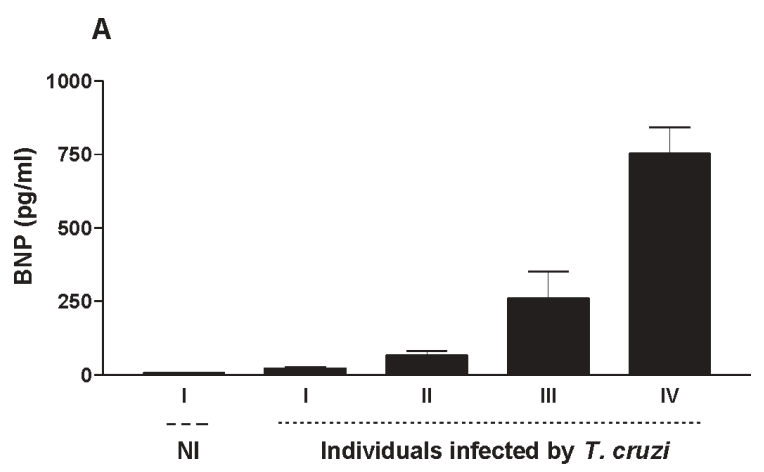

B

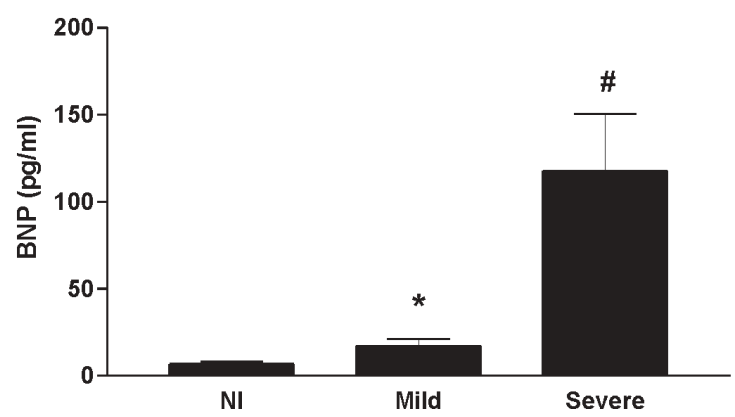

Fig. 1: concentration of brain natriuretic peptide (BNP) in plasma of patients classified according to (A) the New York Heart Association (NYHA) and (B) Chagas disease severity. The concentration of BNP (pg/ml) is given as the mean \pm SEM. A: there were 42 patients in NYHA class I, 15 in class II, 3 in class III, and 3 patents in class IV. B: there were 23 and 40 patients with the mild or severe forms, respectively, of chronic chagasic cardiomyopathy and 18 non-infected individuals. $* \mathrm{P}<0.05$ when compared to non-infected individuals and \# P $<0.05$ when compared to mild chronic chagasic cardiomyopathy.

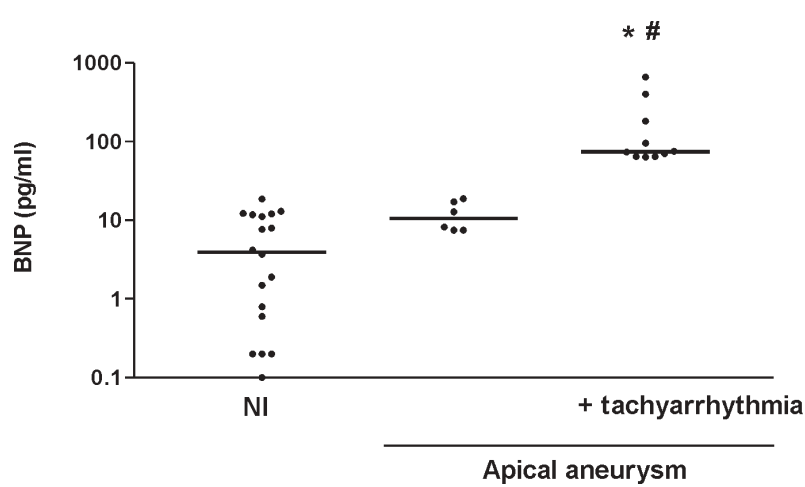

Fig. 2: concentrations of brain natriuretic peptide (BNP) in plasma of chagasic patients with apical aneurysm. $* \mathrm{P}<0.05$ when individuals with apical aneurysm associated with tachyarrhythmia $(\mathrm{n}=$ 10) were compared to non-infected individuals $(n=18)$ and \# P< 0.05 when they were compared to patients with apical lesions alone $(n=6)$. 


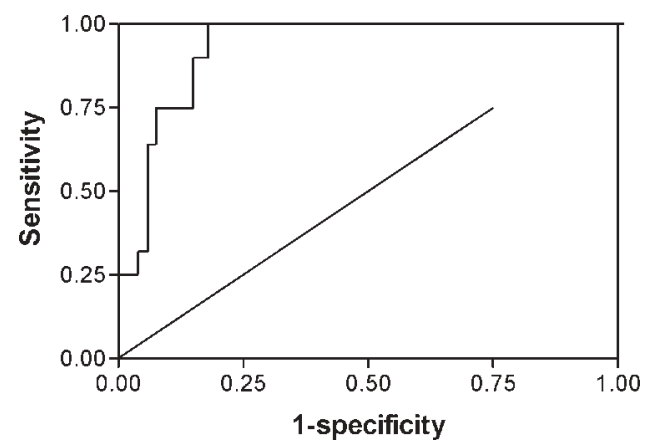

Fig. 3: receiver-operator-characteristic curve for brain natriuretic peptide (BNP) in diagnosis of left ventricular dysfunction. Receiver-operator-characteristic (ROC) curve was assessed for various concentrations of BNP in the diagnosis of left-ventricular dysfunction in the Chagas disease population (area under the ROC curve $=0.92 \pm 0.03$ ).

\section{DISCUSSION}

Natriuretic peptides constitute a system of hormones shown to have diagnostic and prognostic value in patients with congestive heart failure (Clerico et al. 2000, Cheng et al. 2001, Dao et al. 2001, McCullough et al. 2002, de Lemos et al. 2003). Chagasic cardiomyopathy is a quiescent, developmental and complex disease induced in $30-40 \%$ of individuals infected by $T$. cruzi. Affected patients may develop mild, moderate or severe heart disease that will influence in the life span of patients. It is possible that an early diagnosis of heart failure may identify a group of patients that will benefit the most from aggressive medical therapy, although this remains to be determined. Echocardiography is considered the gold standard for the detection of left ventricular dysfunction, but it is relatively costly and not always available in endemic areas where chagasic cardiomyopathy is most common. For this reason, the development of a simple and inexpensive detection method for left ventricular dysfunction in Chagas disease is desirable. At least one study has demonstrated the potential of BNP as a sensitive and specific tool in the diagnosis of heart failure in patients with CCC (Ribeiro et al. 2002b). In the present study, we expand and confirm this previous observation and describe the marked correlation between plasma concentrations of BNP and indices of heart function in chagasic individuals. For the studies described herewith, an internationally acceptable BNP measurement test was used confirming an excellent diagnostic performance in the detection of LV systolic dysfunction (area under the ROC curve was above 0.9). Indeed, the negative predictive value of $98 \%$ indicates that a low BNP value indicates a very low probability of LV dysfunction. Moreover, we confirmed that the ideal cutoff point of BNP levels might vary with the measurement method employed and with the outcome of interest (Ribeiro et al. 2002b, 2003).

The association between BNP concentrations in plasma and the functional NYHA classification of patients was remarkably similar to that already described in other cardiomyopathies (Maeda et al. 1998, Maisel et al. 2002). Moreover, there was a good correlation between concentrations of BNP in plasma and the left ventricle ejection fraction or the left ventricle end-diastolic diameter. Patients with milder forms of Chagas cardiomyopathy showed slightly elevated concentrations of BNP, when compared to controls (see Fig. 1B), confirming that there is a progressive elevation of BNP levels with decreasing left ventricular performance (Ribeiro et al. 2002b). Thus, our studies clearly demonstrate the correlation between BNP levels and the severity of heart failure of chagasic etiology, as it has been demonstrated for heart failure due to other diseases (de Lemos et al. 2001, Maisel et al. 2002, McCullough et al. 2002).

Another interesting finding of our study was the detection of higher concentrations of BNP in patients with apical aneurysm and complex ventricular arrhythmia than in those with apical aneurysm alone. A thinning of the wall localized on the apex of left ventricle (the so called apical lesion), with or without thrombus, is a common finding in Chagas disease, even in patients with less severe forms of CCC (Prata 2001). Complex tachyarrhythmic events are a frequent complication of patients with apical lesions and ventricular dysfunction (Prata 2001). In our study, elevated BNP concentrations were only detected in the latter patients, in whom the ejection fraction was lower. The results once again demonstrate the potential value of BNP in detecting heart failure in chagasic patients and the possible usefulness of BNP measurements in the follow-up of these individuals.

The main stimulus for the increase of BNP synthesis appears to be the stretch of cardiomyocytes by the increased wall stress that occurs in heart failure (Deo \& de Lemos 2003). Of note, the nucleic acid sequence of the BNP gene contains the destabilizing sequence "tatttat", which suggests that the turnover of BNP messenger RNA is high and that BNP is synthesized in bursts, differently from atrial natriuretic peptide, which is synthesized and stored in granules within the atria (Nakagawa et al. 1995, Villacorta et al. 2002). For the latter reasons, BNP has a short half-life, around $22 \mathrm{~min}$ (Maisel et al. 2002), and its determination represents a more dynamic parameter of cardiac function. In such way, it is likely that BNP may not only be useful to detect heart failure, but may also be useful in the follow-up of the response of patients to medical treatment of heart failure, although this remains to be determined in Chagas disease.

In summary, our results suggest that the measurement of the BNP concentration in plasma using a simple, relatively inexpensive and rapid test improves the ability of clinicians to detect heart failure and severe cardiac manifestations in chagasic patients. In the context of Chagas disease, heart failure diagnosis identifies patients with greater risk of severe arrhythmias and sudden death and shorter survival. Such patients may benefit the most from aggressive medical therapy of heart failure. Equally important, patients with $T$. cruzi infection who have a BNP level below $60 \mathrm{pg} / \mathrm{ml}$ appear to have a very low likelihood of severe cardiac involvement, and echocardiography is probably not necessary. 


\section{REFERENCES}

Barros MV, Ribeiro AL, Machado FS, Rocha MOC 2003. Doppler tissue imaging to assess systolic function in Chagas disease. Arq Bras Cardiol 80: 36-40.

Bestetti RB 2001. Predictors of unfavourable prognosis in chronic Chagas' disease. Trop Med Int Health 6: 476-483.

Carrasco HA, Parada H, Guerrero L, Duque M, Duran D, Molina C 1994. Prognostic implications of clinical, electrocardiographic and hemodynamic findings in chronic Chagas' disease. Int J Cardiol 43: 27-38.

Cheng V, Kazanegra R, Garcia A, Lenert L, Krishnaswamy P, Gardetto N, Clopton, P, Maisel A 2001. A rapid bedside test for B-type natriuretic peptide predicts treatment outcomes in patients admitted for decompensated heart failure. A pilot study. J Am Coll Cardiol 37: 386-391.

Clerico A, Del Ry S, Giannessi D 2000. Measurement of cardiac natriuretic hormones (atrial natriuretic peptide, brain natriuretic peptide and related peptides) in clinical practice: the need for a new generation of immunoassay methods. Clin Chem 46: 529-534.

Dao Q, Krishmaswamy P, Kazanegra R, Harrison A, Amirnovin R, Lenert L, Clopton P, Alberto J, Hlavin P, Maisel AS 2001. Utilily of B-type natriuretic peptide in the diagnosis of congestive heart failure in an urgent-care setting. $\mathrm{J} \mathrm{Am}$ Coll Cardiol 37: 370-385.

de Lemos JA, McGuire DK, Drazner MH 2003. B-type Natriuretic Peptide (BNP) in cardiovascular disease. Lancet 362: 316-322.

de Lemos JA, Morrow DA, Bentley JH, Omland T, Sabatine MS, McCabe CH, Hall C, Cannon CP, Braunwald E 2001. The prognostic value of B-type natriuretic peptide in patients with acute coronary syndromes. N Eng J Med 345: 1014-1021.

Deo R, De Lemos JA 2003. B-type natriuretic peptide in ischemic heart disease. Curr Cardiol Rep 5: 271-277.

Kuster GM, Tanner H, Printzen G, Suter TM, Mohacsi P, Hess OM 2003. B-type natriuretic peptide for diagnosis and treatment of congestive heart failure. Swiss Med Wkly 133: 623-628.

Mady C, Cardoso RHA, Barreto ACP, da Luz PL, Bellotti G, Pileggi F 1994. Survival and predictors of survival in patients with congestive heart failure due to Chagas' cardiomyopathy. Circulation 90: 3098-3102.

Maeda K, Takayoshi T, Wada A, Hisanaga T, Kinoshita M 1998. Plasma brain natriuretic peptide as a biochemical marker of high left ventricular end-diastolic pressure in patients with symptomatic left ventricular dysfunction. Am Heart J 135: 825-832.

Maguire JH, Hoff R, Sherlock I, Guimaraes AC, Sleigh AC, Ramos NB, Mott KE, Weller TH 1987. Cardiac morbidity and mortality due to Chagas' disease: prospective electro- cardiographic study of a Brazilian community. Circulation 75: $1140-1145$.

Maisel A, Krishnaswamy P, Nowak RM, McCord J, Hollander JE, Duc P, Omland T, Storrow AB, Abraham WT, Wu AH, Clopton P, Steg PG, Westheim A, Knudsen, CW, Perez A, Kazanegra R, Herrmann HC, McCullough PA 2002. Rapid measurement of B-type natriuretic peptide in the emergency diagnosis of heart failure. N Engl J Med 347: 161167.

McCullough PA, Nowak RM, McCord J, Hollander JE, Herrmann HC, Steg PG, Duc P, Westheim A, Omland T, Knudsen CW, Storrow AB, Abraham WT, Lamba S, Wu AH, Perez A, Clopton P, Krishnaswamy P, Kazanegra R, Maisel AS 2002. B-type natriuretic peptide and clinical judgment in emergency diagnosis of heart failure. Circulation 106: 416-422.

Nakagawa O, Ogawa Y, Itoh H 1995. Rapid transcriptional activation and early mRNA turnover of BNP in cardiocyte hypertrophy: evidence for BNP as an emergency cardiac hormone against ventricular overload. J Clin Invest 96: 1280-1287.

Perez AA, Ribeiro AL, Barros MV, de Sousa MR, Bittencourt RJ, Machado FS, Rocha MO 2003. Value of the radiological study of the thorax for diagnosing left ventricular dysfunction in Chagas' disease. Arq Bras Cardiol 80: 208-213.

Prata A 2001. Clinical and epidemiological aspects of Chagas disease. The Lancet 1: 92-100.

Rassi Jr A, Rassi A, Little WC 2000. Chagas' heart disease. Clin Cardiol 23: 883-889.

Ribeiro AL, Lombardi F, Sousa MR, Barros MV, Porta A, Costa VB, Gomes ME, Santana MF, Rocha MOC 2002a. Powerlaw behavior of heart rate variability in Chagas' disease. Am J Cardiol 89: 414-418.

Ribeiro ALP, Reis AM, Barros MV, Sousa MR, Rocha ALL, Perez AA, Pereira JB, Machado FS, Rocha MOC 2002b. Brain natriuretic peptide in the diagnosis of systolic left ventricular dysfunction in Chagas disease. Lancet 360: 461-462.

Ribeiro AL, Reis AM, Teixeira MM, Rocha MOC 2003. Brain natriuretic peptide in Chagas disease: further insights. Lancet $362: 333$.

Rocha MOC, Ribeiro AL, Teixeira MM 2003. Clinical Managemente of chronic Chagas cardiomyopathy. In Cap. Chagas heart disease. Front Biosc 8: E44-54.

Silberbach M, Roberts CT 2001. Natriuretic peptide signaling molecular and cellular pathways to growth regulation. $\mathrm{Cel}$ lular Signalling 13: 221-231.

Villacorta H, Duarte A, Duarte NM, Carrano A, Mesquista ET, Dohmann HJ, Ferreira FE 2002. The role of B-type natriuretic peptide in the diagnosis of congestive heart failure in patients presenting to an emergency department with dyspnea. Arq Bras Cardiol 79: 569-572. 
